\title{
Review of: "Determinants of COVID-19 vaccine acceptance in a high infection-rate country: a cross- sectional study in Russia"
}

samuel olowookere

Potential competing interests: The author(s) declared that no potential competing interests exist.

Good manuscript 